\title{
Brand-Specific Enhanced Safety Surveillance of GSK's Quadrivalent Seasonal Influenza Vaccine in Belgium, Germany and Spain for the 2018/2019 Season
}

\author{
Gael Dos Santos ${ }^{1}\left[\right.$ - Bach-Yen Nguyen ${ }^{2} \cdot$ Silvia Damaso $^{1}$ (D) - Lode Godderis ${ }^{3,4} \cdot$ Xavier Martínez-Gómez $^{5}$. \\ Tamara Eckermann ${ }^{6} \cdot$ Hugo Loos $^{7} \cdot$ Ignacio Salamanca de la Cueva ${ }^{8} \cdot$ Vishvesh Shende $^{9}$. \\ Alexander C. Schmidt ${ }^{2,10}$ (1) . Anne Yeakey ${ }^{2}$ (1)
}

Published online: 28 December 2019

(c) The Author(s) 2019

\begin{abstract}
Introduction Seasonal influenza causes numerous deaths worldwide each year. Annual vaccination for disease prevention is crucial. Seasonal vaccines are updated each year to closely match circulating strains.

Objective To comply with European Medicines Agency (EMA) guidance, an enhanced safety study was conducted to rapidly collect and assess adverse events (AEs) within 7 days following vaccination with GSK's inactivated quadrivalent seasonal influenza vaccine (IIV4) in 2018/2019.

Methods A customised AE reporting card (AERC) and standardised electronic data reporting application were used in Belgium, Germany and Spain in adult and paediatric subjects in this study.

Results In 2018, 1060 subjects vaccinated with one dose of GSK's IIV4 were enrolled (all subjects in Belgium and Germany were adults, and $75 \%$ and $25 \%$ of subjects in Spain were children and adults, respectively). In Spain, 139 eligible children later received a second dose. Overall 1035 subjects completed the study. After dose 1 and dose 2, 98.3\% and 100\% of subjects, respectively, returned the completed AERC. Over the study period, 43.0\% (456/1060 post dose 1) and 23.7\% (33/139 post dose 2) of subjects reported at least one $\mathrm{AE}$ within 7 days after immunisation. The most frequently reported categories of AEs were General and Administration Site (e.g. injection site pain, swelling, erythema) and Respiratory Disorders (e.g. rhinorrhoea, cough, nasal congestion). There were no deaths and no serious AEs deemed related to GSK's IIV4.

Conclusion In compliance with EMA guidance, this study design allowed for near real-time assessment of AEs. No safety signals were detected at any point during the study period. The study supports and confirms the acceptable safety profile of GSK's IIV4.
\end{abstract}

ClinicalTrials.gov identifier NCT03688620.

\section{Introduction}

Influenza, which occurs in annual epidemics and outbreaks, is a major public health burden responsible for an estimated 290,000-650,000 respiratory deaths each year globally [1]. In the European Union (EU), depending on seasonal variation, the number of symptomatic cases ranges from 4 to 50 million, with 15,000-70,000 deaths each year [2]. Annual vaccination is considered the most effective way to reduce the risk of acquiring influenza and its complications [3]. Due to frequent genetic and antigenic changes in influenza viruses,

Gael Dos Santos

gael.x.dos-santos@gsk.com

Extended author information available on the last page of the article seasonal vaccines are frequently reformulated with updated viral strains based on annual recommendations from the World Health Organization (WHO) [4]. Therefore, there is a need for routine benefit-risk monitoring of seasonal vaccines.

Regularly monitoring for adverse events (AEs) after vaccination is an important component of routine pharmacovigilance and vaccine safety assessments. For several decades, there was a requirement for vaccine manufacturers to conduct small clinical trials with strain-updated seasonal influenza vaccines prior to use in each upcoming season. However, those trials were not considered sufficiently informative, e.g. not large enough to detect the vaccines' expected efficacy and safety. In addition, it was recognised that much safety data existed from surveillance studies and this was unlikely to change significantly with the annual 


\section{Key Points}

The safety of vaccines with annual strains updates need continuous monitoring. The European Medicines Agency (EMA) provides guidelines so vaccine manufacturers can rapidly identify any safety concerns.

To comply with EMA guidance, this enhanced safety study was conducted to rapidly collect and assess adverse events (AEs) following routine vaccination with GSK's inactivated quadrivalent seasonal influenza vaccine in 2018/2019, in children and adults in Belgium, Germany and Spain.

In the 1060 subjects included, $43.0 \%$ and $23.7 \%$ reported at least one AE after dose 1 and dose 2, respectively. No unexpected safety issues were found with GSK's vaccine, which supports and confirms the acceptable safety profile of GSK's IIV4.

updates to seasonal vaccines [5]. The European Medicines Agency (EMA) decided therefore to withdraw this requirement, emphasising instead the need for strengthened and sustainable monitoring of vaccine performance over the years. New guidance on enhanced safety surveillance for seasonal influenza vaccines in the EU was released in 2014 $[6,7]$. The guidance sets out standards for surveillance that all Marketing Authorisation Holders (MAHs) of vaccines in the EU must address. The overarching objective is to rapidly detect any clinically significant increase in the frequency and/or severity of expected reactogenicity (local, systemic or allergic reactions) that may indicate a potential safety concern requiring further investigation.

In order to comply with this requirement, GSK launched annual enhanced safety studies from the 2015/2016 season onwards [8-12], and the final results of the 2018/2019 season study are presented in this manuscript (see published protocol [13]). The countries identified as suitable to host this study were Belgium, Germany, and Spain, where GSK's inactivated quadrivalent seasonal influenza vaccine (IIV4) is distributed (as AlphaRix Tetra, Influsplit Tetra and Fluarix Tetra, respectively) and used in subjects of all ages for which the vaccine is indicated. This study was designed to capture AEs in near real-time using a customised AE reporting card (AERC) and an electronic case report form, in order to rapidly and continuously monitor AEs of interest (AEIs) experienced within 7 days following vaccination.

The primary objective was to estimate, in each country and overall, the cumulative percentage of subjects reporting AEIs and/or other AEs occurring within 7 days of vaccination with GSK's IIV4 using the AERC.

\section{Methods}

\subsection{Study Design and Population}

This was a prospective enhanced safety study of GSK's IIV4 (AlphaRix Tetra in Belgium, Influsplit Tetra in Germany, Fluarix Tetra in Spain-a split virion, inactivated, thimerosal-free, non-adjuvanted vaccine, administered using a prefilled syringe) conducted between 1 October 2018 and 4 January 2019. The aim of the study was to assess safety events occurring after vaccination and thus to contribute to better detecting potential safety signals in near real-time using a customised AERC and electronic reporting system. Predefined AEIs (defined by the EMA to identify common events associated with influenza vaccines) were listed on the AERC, as well as the possibility to report if any other AE or no AE occurred, allowing to obtain a more reliable numerator and denominator to calculate the AEs.

Subjects vaccinated with IIV4 according to routine country-specific practice [14] were enrolled after written informed consent was obtained from the subject or their legally acceptable representative according to local guidelines.

An AERC was given to vaccinated subjects to report any AEs occurring within 7 days of vaccination (i.e. on the day of vaccination and the following 6 days), and was to be returned to their healthcare professional (HCP) at the next scheduled visit or by mail, after which the data were to be entered into the electronic case report form by the medical team.

Approximately 1000 subjects were planned to be enrolled from nine HCPs across Belgium, Germany and Spain, with recruitment planned from 1 October until 31 December for subjects requiring one dose, and until 1 December for subjects requiring two doses 4 weeks apart (i.e. under 9 years of age and not previously vaccinated against influenza) to allow sufficient time for follow-up. In order to include subjects of all ages for which IIV4 is indicated, subjects aged 18 years and above were recruited in Belgium and Germany, where vaccine coverage in children is low (with German sites targeting subjects aged 50 years and older). Recruitment of subjects between 6 months and 65 years of age was targeted in Spain at the time of the vaccination. Children in care were excluded from the study. The study methods have previously been published [13].

\subsection{Statistical Methods}

All analyses were descriptive and are presented by country and overall. All enrolled subjects were vaccinated with GSK's IIV4 and provided written informed consent prior to enrolment. The analysis of AEs was performed on the Safety Set, which included all enrolled subjects who received the AERC to complete. 
Demographic characteristics (age at vaccination [dose 1], sex, geographic ancestry) and risk status for influenzaassociated morbidity and mortality were summarised using frequency tables $[n(\%)]$ for categorical variables, and mean, standard deviation, median, minimum and maximum for continuous data.

The number and percentage of subjects who received a coadministered vaccination on the same day as the GSK's IIV4 were tabulated by vaccination class. The percentage of subjects who received and returned the AERC with a documented presence or absence of AEs were tabulated by centre/country.

For each vaccine dose, the cumulative percentage of subjects reporting AEs from study start up to each study week (i.e. International Standards Organization [ISO] weeks 40-52) was estimated using the Medical Dictionary for Regulatory Activities (MedDRA) [15] Primary System Organ Class (SOC) and Preferred Term (PT). In addition, for each vaccine dose, the weekly and cumulative percentages of subjects reporting AEs were estimated by age strata (6 months-17 years; $18-65$ years; $>65$ years) and risk status (at risk/not at risk) for each country. However, these secondary objectives were not the primary focus and most of them are not presented in detail here. The outcomes for which data are not reported herein can be found at ClinicalTrials. gov and the GSK Study Register (NCT03688620) [16, 17].

The $95 \%$ confidence intervals (CIs) accounting for the clustering effect of centres were computed on all estimated percentages. The design effects and intra-cluster correlation coefficients were also estimated for the cumulative percentage of subjects reporting AEs over the whole study period. In addition, recruitment into the study by sites may have created a clustering effect because participants followed by the same HCP tend to share certain demographic and social characteristics. The design effects and intra-cluster correlation coefficients were therefore also estimated for the cumulative percentage of subjects reporting AEs over the whole study period.

\subsection{Ethical Considerations}

The protocol was approved by the independent Ethics Committee or Institutional Review Board of each study centre and written informed consent was obtained from each participant, with the first subject enrolled at the earliest on 1 October 2018. The study protocol has been published [13] and is also available on GSK's Clinical Study Register (GSK Study ID 207737) [17] and on the United States National Library of Medicine website (www.clinicaltrials.gov; NCT03688620) [16].

\section{Results}

\subsection{Subject Disposition and Characteristics}

Between 5 October 2018 and 30 November 2018, 1060 subjects vaccinated with one dose of GSK's IIV4 were enrolled in Belgium $(n=329)$, Germany $(n=278)$ and Spain $(n=$ 453). Of these, 146 paediatric subjects in Spain were eligible for dose 2, and 139 subsequently received two doses. The total number of doses administered was therefore 1199 (329 in Belgium, 278 in Germany and 592 in Spain). All enrolled subjects received the AERC, and $98.3 \%$ and $100 \%$ returned it after dose 1 and dose 2 , respectively, with documentation of the presence or absence of $\mathrm{AE}(\mathrm{s})$. Overall, 1035 subjects completed the study and 25 were withdrawn for the following reasons: lost to follow-up after dose 1 ( $n=16$ in Belgium, $n=1$ in Spain); not willing to attend a visit after dose 1 ( $n=1$ Germany); and consent withdrawn for dose 2 , of which none were due to an AE ( $n=7$ Spain). One of these subjects had fever at visit 2 (4 weeks after dose 1 and thus unrelated to vaccine administration) and was also withdrawn after visit 2 by the investigator, as the vaccination is contraindicated in cases of fever on the day of vaccination (Fig. 1).

Subjects in Belgium and Germany were all adults (59.6\% and $60.1 \%$, respectively, $>65$ years of age), while, in Spain, $74.8 \%$ were children. There was an even distribution of male and female subjects, and $98.0 \%$ were Caucasian. Overall, $62.0 \%$ of subjects in Belgium, $47.8 \%$ in Germany and $49.2 \%$ in Spain were assessed as at risk for influenza-associated morbidity and mortality by the HCP. Among the children who received dose $2,57.6 \%$ were male, $99.3 \%$ were Caucasian, and $34.5 \%$ were considered at risk for influenzaassociated morbidity and mortality (Table 1).

Other vaccines were coadministered on the same day as dose 1 of GSK's IIV4 in a few subjects in Belgium (3/329, $0.9 \%)$ and Germany $(5 / 278,1.8 \%)$ [e.g. pneumococcal vaccines] compared with 11.0\% (50/453) of subjects in Spain (e.g. hepatitis, pneumococcal and meningococcal vaccines). For dose 2 in Spain, 1.4\% (2/139) of subjects received coadministered vaccines (Table 2 ).

\subsection{Adverse Events}

Figure 2 shows the cumulative number of enrolled subjects by country and week, as well as the proportions reporting at least one $\mathrm{AE}$ within 7 days following vaccination (dark-shaded portion of the bars). Dose 1 of GSK's IIV4 was administered during ISO weeks 40-48, and dose 2 was administered during ISO weeks $46-52$ in Spain. The proportion of individuals reporting at least one AE varied from 42.2 to $47.1 \%$ in Belgium, 32.9 to $36.7 \%$ in Germany and 
Fig. 1 Attrition diagram for the Enhanced Safety Surveillance 2018/2019, in Belgium, Spain and Germany, with subjects enrolled by country and numbers vaccinated with dose 1 and dose 2. * Of 339 enrolled children, 146 were eligible for dose 2 , i.e. receiving the seasonal influenza vaccine for the first time and $\leq 9$ years of age at inclusion. IIV4 inactivated quadrivalent seasonal influenza vaccine, $A E$ adverse event

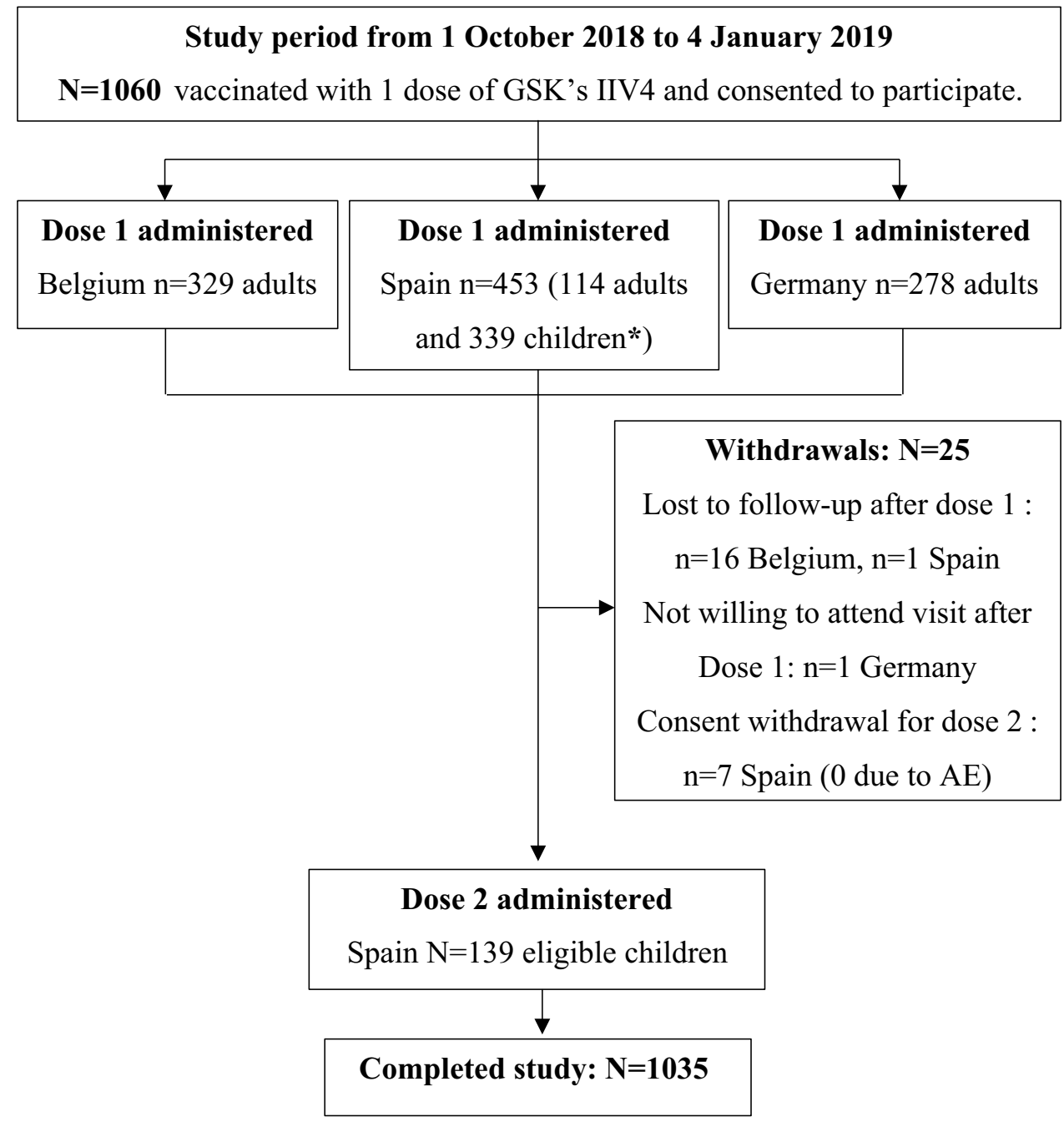

42.6 to $47.5 \%$ in Spain after dose 1, and 23.6 to $45.5 \%$ in Spain after dose 2 (Fig. 2).

Over the study period, overall $43.0 \%$ (456/1060) of subjects reported at least one $\mathrm{AE}$ within 7 days of receiving dose 1 (Table 3). The most frequently reported MedDRA SOCs were General Disorders and Administration Site Conditions in $28.5 \%(302 / 1060)$ of subjects (e.g. injection site pain, swelling or erythema and fatigue) and Respiratory, Thoracic and Mediastinal Disorders in 17.4\% (184/1060) of subjects (e.g. rhinorrhoea, cough, nasal congestion and oropharyngeal pain. Following dose 2, 23.7\% (33/139) of subjects reported at least one AE (Table 4). The most frequently reported MedDRA SOCs were also Respiratory, Thoracic and Mediastinal Disorders in 13.7\% (19/139) of subjects (e.g. rhinorrhoea and cough) and General Disorders and Administration Site Conditions in 9.4\% (13/139) of subjects (e.g. injection site pain and pyrexia). In Spain, following dose 1 or dose 2, overall 50.1\% (227/453) of subjects reported at least one AE within 7 days of vaccination
(Table 5). The most frequently reported MedDRA SOCs were again General Disorders and Administration Site Conditions in $32.2 \%$ (146/453) of subjects (e.g. injection site pain or swelling, pyrexia and fatigue) and Respiratory, Thoracic and Mediastinal Disorders in 25.6\% (116/453) of subjects (e.g. cough, rhinorrhoea and nasal congestion).

The most frequently reported AEs were on the predefined list of AEIs. Following dose 1, these were (by MedDRA PTs) injection site pain (17.6\% of subjects), rhinorrhoea $(8.7 \%)$, injection site swelling $(7.6 \%)$, headache $(7.4 \%)$, myalgia $(6.9 \%)$, cough $(6.7 \%)$, fatigue $(6.5 \%)$, nasal congestion $(5.4 \%)$, injection site erythema $(5.3 \%)$ and oropharyngeal pain (4.8\%). Other AEIs were reported by $\leq 3.4 \%$ of subjects (Table 3). Following dose 2 in paediatric subjects, these were (by MedDRA PTs) cough (9.4\%), rhinorrhoea (7.2\%), injection site pain and pyrexia (5.0\% each). Other AEIs were reported by fewer than $3 \%$ of subjects. AEs not on the predefined list of expected AEIs were reported by 
Table 1 Summary of demographic characteristics and risk status (safety set)

\begin{tabular}{|c|c|c|c|c|c|c|c|c|}
\hline Dose 1 & \multicolumn{2}{|c|}{ Belgium $[N=329]$} & \multicolumn{2}{|c|}{ Germany $[N=278]$} & \multicolumn{2}{|c|}{ Spain $[N=453]$} & \multicolumn{2}{|c|}{ Total $[N=1060]$} \\
\hline \multicolumn{9}{|c|}{ Age at dose 1 vaccination with GSK's IIV4 } \\
\hline Mean (SD) (years) & \multicolumn{2}{|c|}{$61.3(18.2)$} & \multicolumn{2}{|c|}{$65.0(15.1)$} & \multicolumn{2}{|c|}{$15.7(18.7)$} & \multicolumn{2}{|c|}{$42.8(29.4)$} \\
\hline Median (years) & \multicolumn{2}{|c|}{68.0} & \multicolumn{2}{|c|}{68.5} & \multicolumn{2}{|l|}{6.0} & \multicolumn{2}{|l|}{48.0} \\
\hline Minimum age & \multicolumn{2}{|c|}{23 years } & \multicolumn{2}{|c|}{18 years } & \multicolumn{2}{|c|}{6 months } & \multicolumn{2}{|c|}{6 months } \\
\hline \multirow[t]{2}{*}{ Maximum age } & \multicolumn{2}{|c|}{91 years } & \multicolumn{2}{|c|}{100 years } & \multicolumn{2}{|c|}{64 years } & \multicolumn{2}{|c|}{100 years } \\
\hline & $n$ & $\%$ & $n$ & $\%$ & $n$ & $\%$ & $n$ & $\%$ \\
\hline \multicolumn{9}{|l|}{ Age category } \\
\hline 6 months -17 years & 0 & 0.0 & 0 & 0.0 & 339 & 74.8 & 339 & 32.0 \\
\hline $18-65$ years & 133 & 40.4 & 111 & 39.9 & 114 & 25.2 & 358 & 33.8 \\
\hline$>65$ years & 196 & 59.6 & 167 & 60.1 & 0 & 0.0 & 363 & 34.2 \\
\hline Sex & & & & & & & & \\
\hline Female & 147 & 44.7 & 166 & 59.7 & 231 & 51.0 & 544 & 51.3 \\
\hline Male & 182 & 55.3 & 112 & 40.3 & 222 & 49.0 & 516 & 48.7 \\
\hline Geographic ancestry & & & & & & & & \\
\hline $\begin{array}{l}\text { Black or African Ameri- } \\
\text { can }\end{array}$ & 0 & 0.0 & 2 & 0.7 & 1 & 0.2 & 3 & 0.3 \\
\hline Central/South Asian & 1 & 0.3 & 1 & 0.4 & 1 & 0.2 & 3 & 0.3 \\
\hline East Asian & 4 & 1.2 & 1 & 0.4 & 0 & 0.0 & 5 & 0.5 \\
\hline Southeast Asian & 2 & 0.6 & 0 & 0.0 & 0 & 0.0 & 2 & 0.2 \\
\hline $\begin{array}{l}\text { White-Arabic/North } \\
\text { African }\end{array}$ & 1 & 0.3 & 0 & 0.0 & 3 & 0.7 & 4 & 0.4 \\
\hline Caucasian/European & 321 & 97.6 & 274 & 98.6 & 444 & 98.0 & 1039 & 98.0 \\
\hline Other & 0 & 0.0 & 0 & 0.0 & 4 & 0.9 & 4 & 0.4 \\
\hline Risk status for influenza & associ & orbidity & lortal & & & & & \\
\hline At risk & 204 & 62.0 & 133 & 47.8 & 223 & 49.2 & 560 & 52.8 \\
\hline Not at risk & 125 & 38.0 & 145 & 52.2 & 230 & 50.8 & 500 & 47.2 \\
\hline Dose 2 & & & & & Spai & 139] & Tota & $=139]$ \\
\hline Age at dose 1 vaccinatio & with & IIV4 & & & & & & \\
\hline Mean (SD) (years) & & & & & 2.9 & & 2.9( & \\
\hline Median (years) & & & & & 3.0 & & 3.0 & \\
\hline Minimum age (months) & & & & & 6 & & 6 & \\
\hline Maximum age (years) & & & & & 8 & & 8 & \\
\hline & & & & & $n$ & $\%$ & $n$ & $\%$ \\
\hline Age category & & & & & & & & \\
\hline 6 months -17 years & & & & & 139 & 100 & 139 & 100 \\
\hline Sex & & & & & & & & \\
\hline Female & & & & & 59 & 42.4 & 59 & 42.4 \\
\hline Male & & & & & 80 & 57.6 & 80 & 57.6 \\
\hline Geographic ancestry & & & & & & & & \\
\hline Central/South Asian & & & & & 1 & 0.7 & 1 & 0.7 \\
\hline Caucasian/European & & & & & 138 & 99.3 & 138 & 99.3 \\
\hline Risk status for influenza & associ & orbidity & cortal & & & & & \\
\hline At risk & & & & & 48 & 34.5 & 48 & 34.5 \\
\hline Not at risk & & & & & 91 & 65.5 & 91 & 65.5 \\
\hline
\end{tabular}

IIV4 inactivated quadrivalent seasonal influenza vaccine, $N$ number of subjects, $n / \%$ number/percentage of subjects in the category, $S D$ standard deviation

${ }^{a}$ Assessed by the healthcare professional based on his/her judgement and experience 
Table 2 Vaccines coadministered with GSK's IIV4

\begin{tabular}{|c|c|c|c|c|c|c|c|c|c|}
\hline \multirow[t]{2}{*}{ GSK IIV4 } & \multirow{2}{*}{$\begin{array}{l}\text { Coadministered vaccine } \\
\text { class }^{\mathrm{a}}\end{array}$} & \multicolumn{2}{|c|}{ Belgium $[N=329]$} & \multicolumn{2}{|c|}{ Germany $[N=278]$} & \multicolumn{2}{|c|}{ Spain $[N=453]$} & \multicolumn{2}{|c|}{ Total $[N=1060]$} \\
\hline & & $n$ & $\%$ & $n$ & $\%$ & $n$ & $\%$ & $n$ & $\%$ \\
\hline \multirow[t]{11}{*}{ Dose 1} & Any & 3 & 0.9 & 5 & 1.8 & 50 & 11.0 & 58 & 5.5 \\
\hline & Bacterial + viral & & & & & 2 & 0.4 & 2 & 0.2 \\
\hline & Hib & & & & & 2 & 0.4 & 2 & 0.2 \\
\hline & Hepatitis & & & & & 21 & 4.6 & 21 & 2.0 \\
\hline & Meningococcal & & & & & 13 & 2.9 & 13 & 1.2 \\
\hline & Multiple & & & 2 & 0.7 & & & 2 & 0.2 \\
\hline & Other viral & & & & & 6 & 1.3 & 6 & 0.6 \\
\hline & Pneumococcal & 3 & 0.9 & 3 & 1.1 & 18 & 4.0 & 24 & 2.3 \\
\hline & Poliomyelitis & & & & & 1 & 0.2 & 1 & 0.1 \\
\hline & Varicella zoster & & & & & 1 & 0.2 & 1 & 0.1 \\
\hline & & & & & & \multicolumn{2}{|c|}{ Spain $[N=139]$} & \multicolumn{2}{|c|}{ Total $[N=139]$} \\
\hline \multirow[t]{3}{*}{ Dose 2} & Any & & & & & 2 & 1.4 & 2 & 1.4 \\
\hline & Hepatitis & & & & & 1 & 0.7 & 1 & 0.7 \\
\hline & $\begin{array}{l}\text { Meningo- } \\
\text { coccal }\end{array}$ & & & & & 1 & 0.7 & 1 & 0.7 \\
\hline
\end{tabular}

Hib Haemophilus influenzae B, IIV4 inactivated quadrivalent seasonal influenza vaccine, $N$ number of subjects vaccinated with GSK's IIV4, $n$ number of subjects with coadministered vaccine

${ }^{\mathrm{a}}$ Vaccination classes coded using the GSK Drug dictionary

Fig. 2 The cumulative number of enrolled subjects by week and country is presented, showing, in darker shades, the proportion reporting at least one $\mathrm{AE}$ (percentage with AEs are indicated in the coloured fields below the histogram). Subjects in Germany and Belgium were adults and therefore received only one dose of GSK's inactivated quadrivalent influenza vaccine, while some subjects in Spain were children eligible for two doses. $A E$ adverse event

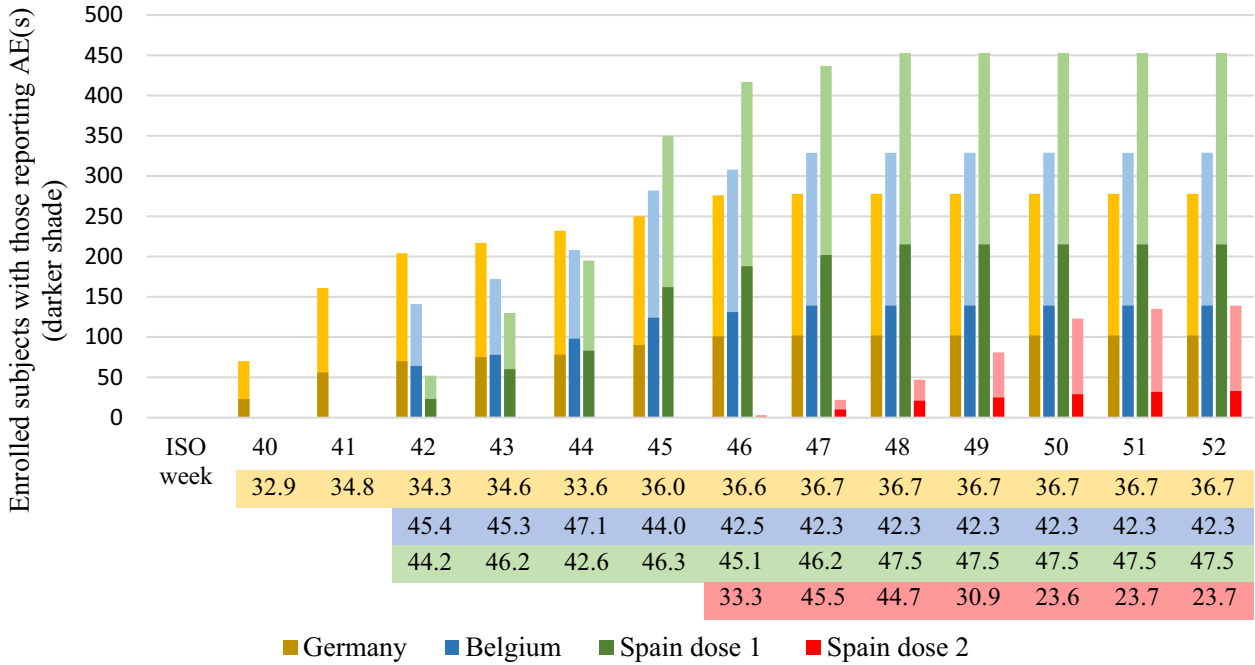

The proportion of subjects reporting AEs regardless of risk factors for influenza-associated morbidity and mortality was similar: $43.2 \%$ (95\% CI 33.7-53.2 with risk) versus $42.8 \%$ (95\% CI 37.6-48.1 without risk) after dose 1, and $29.2 \%$ (95\% CI 1.1-83.9 with risk) versus $20.9 \%$ (95\% CI 8.2-39.8 without risk) after dose 2. By age category (6 months-17 years; $18-65$ years; $>65$ years) post dose 1 , the cumulative percentage of subjects reporting any AEs was highest among adults $(49.2 \%, 95 \%$ CI 42.0-56.4), followed by children $(45.7 \%, 95 \%$ CI $20.1-73.2)$ and older adults (34.4\%, 95\% CI 25.3-44.6). 
Table 3 Cumulative subjects (\%) reporting AEIs (italic) and/or other AEs post dose 1 over the study period (Safety Set)

\begin{tabular}{|c|c|c|c|c|c|}
\hline $\begin{array}{l}\text { MedDRA Primary System Organ } \\
\text { Class (CODE) } \\
\text { Preferred Term (Code) }\end{array}$ & & Belgium $[N=329]$ & Germany $[N=278]$ & Spain $[N=453]$ & Total $[N=1060]$ \\
\hline Any & $\begin{array}{l}\text { n }(\%) \\
\text { 95\% CI, } \\
\text { LL-UL }\end{array}$ & $\begin{array}{l}139(42.3) \\
22.2-64.3\end{array}$ & $\begin{array}{l}102(36.7) \\
15.8-62.0\end{array}$ & $\begin{array}{l}215(47.5) \\
23.2-72.6\end{array}$ & $\begin{array}{l}456(43.0) \\
36.8-49.4\end{array}$ \\
\hline $\begin{array}{l}\text { Any general disorders and } \\
\text { administration site conditions } \\
(\mathbf{1 0 0 1 8 0 6 5 )}\end{array}$ & $\begin{array}{l}\text { n }(\%) \\
\text { 95\% CI, } \\
\text { LL-UL }\end{array}$ & $\begin{array}{l}88(26.8) \\
6.3-59.0\end{array}$ & $\begin{array}{l}77(27.7) \\
14.7-44.2\end{array}$ & $\begin{array}{l}137(30.2) \\
7.3-64.2\end{array}$ & $\begin{array}{l}302(28.5) \\
21.8-35.9\end{array}$ \\
\hline Injection site pain (10022086) & & $\begin{array}{l}65(19.8) \\
1.3-62.8\end{array}$ & $\begin{array}{l}46(16.6) \\
5.7-34.1\end{array}$ & $\begin{array}{l}76(16.8) \\
1.0-56.7\end{array}$ & $\begin{array}{l}187(17.6) \\
11.2-25.8\end{array}$ \\
\hline Injection site swelling (10053425) & & $\begin{array}{l}17(5.2) \\
3.0-8.1\end{array}$ & $\begin{array}{l}30(10.8) \\
7.3-15.2\end{array}$ & $\begin{array}{l}33(7.3) \\
1.7-18.9\end{array}$ & $\begin{array}{l}80(7.6) \\
5.4-10.2\end{array}$ \\
\hline Fatigue (10016256) & & $\begin{array}{l}13(4.0) \\
0.6-12.7\end{array}$ & $\begin{array}{l}22(7.9) \\
2.3-18.5\end{array}$ & $\begin{array}{l}34(7.5) \\
1.2-22.8\end{array}$ & $\begin{array}{l}69(6.5) \\
4.0-9.9\end{array}$ \\
\hline Injection site erythema (10022061) & & $\begin{array}{l}10(3.0) \\
0.0-18.7\end{array}$ & $\begin{array}{l}21(7.6) \\
1.5-21.3\end{array}$ & $\begin{array}{l}25(5.5) \\
2.4-10.6\end{array}$ & $\begin{array}{l}56(5.3) \\
3.5-7.6\end{array}$ \\
\hline Pyrexia (10037660) & & $\begin{array}{l}2(0.6) \\
0.0-6.0\end{array}$ & $\begin{array}{l}2(0.7) \\
0.0-14.0\end{array}$ & $\begin{array}{l}29(6.4) \\
3.0-11.8\end{array}$ & $\begin{array}{l}33(3.1) \\
0.9-7.5\end{array}$ \\
\hline Chills (10008531) & & $\begin{array}{l}5(1.5) \\
0.0-7.9\end{array}$ & $\begin{array}{l}7(2.5) \\
0.0-28.3\end{array}$ & $\begin{array}{l}13(2.9) \\
0.3-10.1\end{array}$ & $\begin{array}{l}25(2.4) \\
1.1-4.4\end{array}$ \\
\hline Injection site pruritus (10022093) & & $\begin{array}{l}1(0.3) \\
0.0-3.9\end{array}$ & $\begin{array}{l}3(1.1) \\
0.0-13.1\end{array}$ & $\begin{array}{l}1(0.2) \\
0.0-4.8\end{array}$ & $\begin{array}{l}5(0.5) \\
0.1-1.7\end{array}$ \\
\hline Face oedema (10016029) & & $\begin{array}{l}1(0.3) \\
0.0-9.8\end{array}$ & $\begin{array}{l}0(0.0) \\
0.0-1.3\end{array}$ & $\begin{array}{l}1(0.2) \\
0.0-2.3\end{array}$ & $\begin{array}{l}2(0.2) \\
0.0-0.7\end{array}$ \\
\hline $\begin{array}{l}\text { Influenza-like illness (10022004); } \\
\text { mucous membrane disorder } \\
\text { (10028133); peripheral swelling } \\
\text { (10048959) }\end{array}$ & & $\begin{array}{l}n=1 ; 0 ; 1 \\
\mathrm{NR}\end{array}$ & $\begin{array}{l}n=0 ; 0 ; 0 \\
\text { NR }\end{array}$ & $\begin{array}{l}n=1 ; 2 ; 1 \\
\mathrm{NR}\end{array}$ & $\begin{array}{l}2(0.2) \text { each } \\
\text { NR }\end{array}$ \\
\hline $\begin{array}{l}\text { Discomfort (10013082); feel- } \\
\text { ing cold (10016326); feeling } \\
\text { hot (10016334); injection site } \\
\text { hematoma (10022066); injection } \\
\text { site warmth (10022112); malaise } \\
\text { (10025482) }\end{array}$ & & $\begin{array}{l}n=0 ; 1 ; 1 ; 0 ; 1 ; 0 \\
\mathrm{NR}\end{array}$ & $\begin{array}{l}n=0 ; 0 ; 0 ; 1 ; 0 ; 1 \\
\mathrm{NR}\end{array}$ & $\begin{array}{l}n=1 ; 0 ; 0 ; 0 ; 0 ; 0 \\
\text { NR }\end{array}$ & $\begin{array}{l}1(0.1) \text { each } \\
\text { NR }\end{array}$ \\
\hline $\begin{array}{l}\text { Any respiratory, thoracic and } \\
\text { mediastinal disorders }(\mathbf{1 0 0 3 8 7 3 8})\end{array}$ & $\begin{array}{l}\text { n }(\%) \\
\text { 95\% CI, } \\
\text { LL-UL }\end{array}$ & $\begin{array}{l}48(14.6) \\
4.0-33.6\end{array}$ & $\begin{array}{l}32(11.5) \\
3.0-27.6\end{array}$ & $\begin{array}{l}104(23.0) \\
12.9-36.0\end{array}$ & $\begin{array}{l}184(17.4) \\
12.6-23.0\end{array}$ \\
\hline Rhinorrhoea (10039101) & & $\begin{array}{l}30(9.1) \\
0.2-42.6\end{array}$ & $\begin{array}{l}15(5.4) \\
2.2-10.9\end{array}$ & $\begin{array}{l}47(10.4) \\
4.5-19.6\end{array}$ & $\begin{array}{l}92(8.7) \\
5.8-12.4\end{array}$ \\
\hline Cough (10011224) & & $\begin{array}{l}12(3.7) \\
1.9-6.3\end{array}$ & $\begin{array}{l}11(4.0) \\
0.4-14.7\end{array}$ & $\begin{array}{l}48(10.6) \\
3.5-23.2\end{array}$ & $\begin{array}{l}71(6.7) \\
3.5-11.4\end{array}$ \\
\hline Nasal congestion (10028735) & & $\begin{array}{l}10(3.0) \\
0.3-11.2\end{array}$ & $\begin{array}{l}10(3.6) \\
0.2-16.3\end{array}$ & $\begin{array}{l}37(8.2) \\
5.8-11.1\end{array}$ & $\begin{array}{l}57(5.4) \\
3.0-8.8\end{array}$ \\
\hline Oropharyngeal pain (10068319) & & $\begin{array}{l}10(3.0) \\
1.1-6.6\end{array}$ & $\begin{array}{l}15(5.4) \\
0.8-17.2\end{array}$ & $\begin{array}{l}26(5.7) \\
1.3-15.2\end{array}$ & $\begin{array}{l}51(4.8) \\
3.2-7.0\end{array}$ \\
\hline Dysphonia (10013952) & & $\begin{array}{l}4(1.2) \\
0.3-3.1\end{array}$ & $\begin{array}{l}9(3.2) \\
0.4-11.0\end{array}$ & $\begin{array}{l}10(2.2) \\
1.1-4.0\end{array}$ & $\begin{array}{l}23(2.2) \\
1.4-3.3\end{array}$ \\
\hline Epistaxis (10015090) & & $\begin{array}{l}1(0.3) \\
0.0-3.9\end{array}$ & $\begin{array}{l}4(1.4) \\
0.1-6.7\end{array}$ & $\begin{array}{l}4(0.9) \\
0.0-7.4\end{array}$ & $\begin{array}{l}9(0.9) \\
0.3-1.9\end{array}$ \\
\hline Wheezing (10047924) & & $\begin{array}{l}1(0.3) \\
0.0-5.5\end{array}$ & $\begin{array}{l}3(1.1) \\
0.0-13.1\end{array}$ & $\begin{array}{l}2(0.4) \\
0.1-1.6\end{array}$ & $\begin{array}{l}6(0.6) \\
0.1-1.5\end{array}$ \\
\hline $\begin{array}{l}\text { Dyspnoea (10013968); sneezing } \\
(10041232)\end{array}$ & & $\begin{array}{l}n=0 ; 1 \\
\mathrm{NR}\end{array}$ & $\begin{array}{l}n=3 ; 0 \\
\mathrm{NR}\end{array}$ & $\begin{array}{l}n=0 ; 2 \\
\mathrm{NR}\end{array}$ & $\begin{array}{l}3(0.3) \text { each } \\
\text { NR }\end{array}$ \\
\hline
\end{tabular}


Table 3 (continued)

\begin{tabular}{|c|c|c|c|c|c|}
\hline \multicolumn{2}{|l|}{$\begin{array}{l}\text { MedDRA Primary System Organ } \\
\text { Class (CODE) } \\
\text { Preferred Term (Code) }\end{array}$} & Belgium $[N=329]$ & Germany $[N=278]$ & Spain $[N=453]$ & Total $[N=1060]$ \\
\hline $\begin{array}{l}\text { Dry throat (10013789); increased } \\
\text { upper airway secretion } \\
\text { (10062717); pharyngeal inflamma- } \\
\text { tion }(10065716) \text {; throat clearing } \\
(10080125)\end{array}$ & & $\begin{array}{l}n=1 ; 0 ; 0 \\
\mathrm{NR}\end{array}$ & $\begin{array}{l}n=0 ; 0 ; 0 \\
\mathrm{NR}\end{array}$ & $\begin{array}{l}n=0 ; 1 ; 1 \\
\mathrm{NR}\end{array}$ & $\begin{array}{l}1(0.1) \text { each } \\
\text { NR }\end{array}$ \\
\hline $\begin{array}{l}\text { Any musculoskeletal and connec- } \\
\text { tive tissue disorders (10028395) }\end{array}$ & $\begin{array}{l}\text { n }(\%) \\
\text { 95\% CI, } \\
\text { LL-UL }\end{array}$ & $\begin{array}{l}35(10.6) \\
3.6-22.9\end{array}$ & $\begin{array}{l}23(8.3) \\
1.5-23.6\end{array}$ & $\begin{array}{l}38(8.4) \\
0.3-37.2\end{array}$ & $\begin{array}{l}96(9.1) \\
5.8-13.3\end{array}$ \\
\hline Myalgia (10028411) & & $\begin{array}{l}27(8.2) \\
1.6-22.6\end{array}$ & $\begin{array}{l}15(5.4) \\
0.6-19.0\end{array}$ & $\begin{array}{l}31(6.8) \\
0.4-28.3\end{array}$ & $\begin{array}{l}73(6.9) \\
4.3-10.3\end{array}$ \\
\hline Arthropathy (10003285) & & $\begin{array}{l}11(3.3) \\
0.2-15.3\end{array}$ & $\begin{array}{l}10(3.6) \\
0.3-13.5\end{array}$ & $\begin{array}{l}11(2.4) \\
0.0-29.2\end{array}$ & $\begin{array}{l}32(3.0) \\
1.3-6.0\end{array}$ \\
\hline Pain in extremity (10033425) & & $\begin{array}{l}0(0.0) \\
0.0-1.1\end{array}$ & $\begin{array}{l}2(0.7) \\
0.1-2.6\end{array}$ & $\begin{array}{l}1(0.2) \\
0.0-2.3\end{array}$ & $\begin{array}{l}3(0.3) \\
0.1-0.8\end{array}$ \\
\hline Rheumatic disorder (10072736) & & $\begin{array}{l}0(0.0) \\
0.0-1.1\end{array}$ & $\begin{array}{l}2(0.7) \\
0.0-8.9\end{array}$ & $\begin{array}{l}0(0.0) \\
0.0-0.8\end{array}$ & $\begin{array}{l}2(0.2) \\
0.0-1.3\end{array}$ \\
\hline $\begin{array}{l}\text { Limb discomfort (10061224); mus- } \\
\text { cle spasms (10028334); musculo- } \\
\text { skeletal pain (10028391); synovial } \\
\text { cyst (10042858) }\end{array}$ & & $\begin{array}{l}n=0 ; 0 ; 0 \\
\mathrm{NR}\end{array}$ & $\begin{array}{l}n=1 ; 1 ; 0 \\
\mathrm{NR}\end{array}$ & $\begin{array}{l}n=0 ; 0 ; 1 \\
\text { NR }\end{array}$ & $\begin{array}{l}1(0.1) \text { each } \\
\text { NR }\end{array}$ \\
\hline $\begin{array}{l}\text { Any nervous system disorders } \\
(\mathbf{1 0 0 2 9 2 0 5 )}\end{array}$ & $\begin{array}{l}\text { n }(\%) \\
\text { 95\% CI, } \\
\text { LL-UL }\end{array}$ & $\begin{array}{l}27(8.2) \\
1.6-22.6\end{array}$ & $\begin{array}{l}22(7.9) \\
1.8-21.0\end{array}$ & $\begin{array}{l}36(8.0) \\
0.6-29.7\end{array}$ & $\begin{array}{l}85(8.0) \\
5.3-11.5\end{array}$ \\
\hline Headache (10019211) & & $\begin{array}{l}24(7.3) \\
1.7-19.2\end{array}$ & $\begin{array}{l}21(7.6) \\
1.3-22.4\end{array}$ & $\begin{array}{l}33(7.3) \\
1.1-22.5\end{array}$ & $\begin{array}{l}78(7.4) \\
5.1-10.2\end{array}$ \\
\hline Dizziness (10013573) & & $\begin{array}{l}3(0.9) \\
0.0-11.3\end{array}$ & $\begin{array}{l}0(0.0) \\
0.0-1.3\end{array}$ & $\begin{array}{l}1(0.2) \\
0.0-4.8\end{array}$ & $\begin{array}{l}4(0.4) \\
0.0-1.7\end{array}$ \\
\hline $\begin{array}{l}\text { Aphonia (10002953); poor quality } \\
\text { sleep (10062519); somnolence } \\
\text { (10041349); tremor (10044565) }\end{array}$ & & $\begin{array}{l}n=0 ; 0 ; 0 ; 1 \\
\text { NR }\end{array}$ & $\begin{array}{l}n=0 ; 1 ; 0 ; 0 \\
\text { NR }\end{array}$ & $\begin{array}{l}n=1 ; 0 ; 1 ; 0 \\
\mathrm{NR}\end{array}$ & $\begin{array}{l}1(0.1) \text { each } \\
\text { NR }\end{array}$ \\
\hline Febrile convulsion (10016284) & & $\begin{array}{l}0(0.0) \\
0.0-1.1\end{array}$ & $\begin{array}{l}0(0.0) \\
0.0-1.3\end{array}$ & $\begin{array}{l}0(0.0) \\
0.0-0.8\end{array}$ & $\begin{array}{l}\text { 0 (0.0) } \\
0.0-0.4\end{array}$ \\
\hline $\begin{array}{l}\text { Any gastrointestinal disorders } \\
\text { (10017947) }\end{array}$ & $\begin{array}{l}\text { n }(\%) \\
\text { 95\% CI, } \\
\text { LL-UL }\end{array}$ & $\begin{array}{l}22(6.7) \\
0.1-33.1\end{array}$ & $\begin{array}{l}14(5.0) \\
0.1-25.5\end{array}$ & $\begin{array}{l}31(6.8) \\
4.1-10.7\end{array}$ & $\begin{array}{l}67(6.3) \\
4.2-9.0\end{array}$ \\
\hline Diarrhoea (10012735) & & $\begin{array}{l}6(1.8) \\
0.0-14.0\end{array}$ & $\begin{array}{l}9(3.2) \\
0.4-11.0\end{array}$ & $\begin{array}{l}18(4.0) \\
2.4-6.2\end{array}$ & $\begin{array}{l}33(3.1) \\
2.0-4.6\end{array}$ \\
\hline Nausea (10028813) & & $\begin{array}{l}14(4.3) \\
0.2-19.1\end{array}$ & $\begin{array}{l}5(1.8) \\
0.0-21.0\end{array}$ & $\begin{array}{l}13(2.9) \\
\mathbf{0 . 3}-\mathbf{1 0 . 1}\end{array}$ & $\begin{array}{l}32(3.0) \\
1.6-5.2\end{array}$ \\
\hline Vomiting (10047700) & & $\begin{array}{l}5(1.5) \\
0.3-4.8\end{array}$ & $\begin{array}{l}0(0.0) \\
0.0-1.3\end{array}$ & $\begin{array}{l}14(3.1) \\
0.4-10.9\end{array}$ & $\begin{array}{l}19(1.8) \\
0.7-3.7\end{array}$ \\
\hline Abdominal pain (10000081) & & $\begin{array}{l}0(0.0) \\
0.0-1.1\end{array}$ & $\begin{array}{l}0(0.0) \\
0.0-1.3\end{array}$ & $\begin{array}{l}3(0.7) \\
0.0-6.9\end{array}$ & $\begin{array}{l}3(0.3) \\
0.0-1.6\end{array}$ \\
\hline $\begin{array}{l}\text { Abdominal discomfort (10000059), } \\
\text { gingival bleeding (10018276); lip } \\
\text { swelling (10024570); stomatitis } \\
\text { (10042128); tongue discomfort } \\
\text { (10077855) }\end{array}$ & & $\begin{array}{l}n=0 ; 0 ; 0 ; 0 ; 0 \\
\mathrm{NR}\end{array}$ & $\begin{array}{l}n=0 ; 1 ; 1 ; 1 ; 1 \\
\mathrm{NR}\end{array}$ & $\begin{array}{l}n=1 ; 0 ; 0 ; 0 ; 0 \\
\mathrm{NR}\end{array}$ & $\begin{array}{l}1(0.1) \text { each } \\
\text { NR }\end{array}$ \\
\hline $\begin{array}{l}\text { Any infections and infestations } \\
(\mathbf{1 0 0 2 1 8 8 1})\end{array}$ & $\begin{array}{l}\text { n }(\%) \\
\text { 95\% CI, } \\
\text { LL-UL }\end{array}$ & $\begin{array}{l}15(4.6) \\
0.1-25.5\end{array}$ & $\begin{array}{l}22(7.9) \\
2.2-19.1\end{array}$ & $\begin{array}{l}29(6.4) \\
0.2-29.1\end{array}$ & $\begin{array}{l}66(6.2) \\
3.5-10.1\end{array}$ \\
\hline Conjunctivitis (10010741) & & $\begin{array}{l}12(3.7) \\
0.1-19.5\end{array}$ & $\begin{array}{l}11(4.0) \\
0.6-12.4\end{array}$ & $\begin{array}{l}13(2.9) \\
0.0-22.6\end{array}$ & $\begin{array}{l}36(3.4) \\
1.6-6.2\end{array}$ \\
\hline Rhinitis (10039083) & & $\begin{array}{l}6(1.8) \\
0.1-8.0\end{array}$ & $\begin{array}{l}13(4.7) \\
0.6-15.4\end{array}$ & $\begin{array}{l}13(2.9) \\
0.1-15.2\end{array}$ & $\begin{array}{l}32(3.0) \\
1.5-5.3\end{array}$ \\
\hline
\end{tabular}


Table 3 (continued)

\begin{tabular}{|c|c|c|c|c|c|}
\hline $\begin{array}{l}\text { MedDRA Primary System Organ } \\
\text { Class (CODE) } \\
\text { Preferred Term (Code) }\end{array}$ & & Belgium $[N=329]$ & Germany $[N=278]$ & Spain $[N=453]$ & Total $[N=1060]$ \\
\hline $\begin{array}{l}\text { Bronchitis }(10006451) \text {; ear infec- } \\
\text { tion (10014011); herpes sim- } \\
\text { plex (10019948); hordeolum } \\
\text { (10020377); laryngitis }(10023874) \text {; } \\
\text { nasopharyngitis }(10028810) \text {, oral } \\
\text { herpes (10067152); upper respira- } \\
\text { tory tract infection (10046306) }\end{array}$ & & $\begin{array}{l}n=0 ; 0 ; 0 ; 0 ; 0 ; 0 ; 0 ; 0 \\
\mathrm{NR}\end{array}$ & $\begin{array}{l}n=0 ; 0 ; 1 ; 0 ; 0 ; 0 ; 1 ; 0 \\
\mathrm{NR}\end{array}$ & $\begin{array}{l}n=1 ; 1 ; 0 ; 1 ; 1 ; 1 ; 0 ; 1 \\
\mathrm{NR}\end{array}$ & $\begin{array}{l}1(0.1) \text { each } \\
\text { NR }\end{array}$ \\
\hline $\begin{array}{l}\text { Any metabolism and nutrition } \\
\text { disorders }(10027433)\end{array}$ & $\begin{array}{l}\text { n }(\%) \\
\text { 95\% CI, } \\
\text { LL-UL }\end{array}$ & $\begin{array}{l}7(2.1) \\
0.1-9.8\end{array}$ & $\begin{array}{l}1(0.4) \\
0.0-7.2\end{array}$ & $\begin{array}{l}24(5.3) \\
0.3-22.7\end{array}$ & $\begin{array}{l}32(3.0) \\
0.8-7.6\end{array}$ \\
\hline Decreased appetite (10061428) & & $\begin{array}{l}7(2.1) \\
0.1-9.8\end{array}$ & $\begin{array}{l}1(0.4) \\
0.0-7.2\end{array}$ & $\begin{array}{l}24(5.3) \\
0.3-22.7\end{array}$ & $\begin{array}{l}32(3.0) \\
0.8-7.6\end{array}$ \\
\hline $\begin{array}{l}\text { Any psychiatric disorders } \\
(\mathbf{1 0 0 3 7 1 7 5 )}\end{array}$ & $\begin{array}{l}\text { n }(\%) \\
\text { 95\% CI, } \\
\text { LL-UL }\end{array}$ & $\begin{array}{l}3(0.9) \\
0.0-7.2\end{array}$ & $\begin{array}{l}5(1.8) \\
0.0-21.0\end{array}$ & $\begin{array}{l}11(2.4) \\
0.4-7.6\end{array}$ & $\begin{array}{l}19(1.8) \\
0.8-3.5\end{array}$ \\
\hline Irritability (10022998) & & $\begin{array}{l}3(0.9) \\
0.0-7.2\end{array}$ & $\begin{array}{l}4(1.4) \\
0.0-17.1\end{array}$ & $\begin{array}{l}11(2.4) \\
0.4-7.6\end{array}$ & $\begin{array}{l}18(1.7) \\
0.7-3.3\end{array}$ \\
\hline $\begin{array}{l}\text { Insomnia (10022437); mood swings } \\
\text { (10027951); sleep disorder } \\
(10040984)\end{array}$ & & $\begin{array}{l}n=0 ; 0 ; 0 \\
\mathrm{NR}\end{array}$ & $\begin{array}{l}n=1 ; 0 ; 1 \\
\text { NR }\end{array}$ & $\begin{array}{l}n=0 ; 1 ; 0 \\
\text { NR }\end{array}$ & $\begin{array}{l}1(0.1) \text { each } \\
\text { NR }\end{array}$ \\
\hline $\begin{array}{l}\text { Any skin and subcutaneous tissue } \\
\text { disorders }(\mathbf{1 0 0 4 0 7 8 5 )}\end{array}$ & $\begin{array}{l}\text { n }(\%) \\
\text { 95\% CI, } \\
\text { LL-UL }\end{array}$ & $\begin{array}{l}6(1.8) \\
0.0-15.6\end{array}$ & $\begin{array}{l}6(2.2) \\
0.3-7.4\end{array}$ & $\begin{array}{l}6(1.3) \\
0.0-11.5\end{array}$ & $\begin{array}{l}18(1.7) \\
0.7-3.4\end{array}$ \\
\hline Pruritus (10037087) & & $\begin{array}{l}3(0.9) \\
0.0-7.2\end{array}$ & $\begin{array}{l}5(1.8) \\
0.0-10.2\end{array}$ & $\begin{array}{l}0(0.0) \\
0.0-0.8\end{array}$ & $\begin{array}{l}8(0.8) \\
0.1-2.4\end{array}$ \\
\hline Rash (10037844) & & $\begin{array}{l}1(0.3) \\
0.0-3.9\end{array}$ & $\begin{array}{l}1(0.4) \\
0.0-7.2\end{array}$ & $\begin{array}{l}4(0.9) \\
0.0-7.8\end{array}$ & $\begin{array}{l}6(0.6) \\
0.2-1.4\end{array}$ \\
\hline $\begin{array}{l}\text { Erythema (10015150), pruritus } \\
\text { generalized (10052576) }\end{array}$ & & $0 ; 2$ & $0 ; 0$ & $2 ; 0$ & $2(0.2)$ each \\
\hline Rash generalized (10037858) & & $\begin{array}{l}0(0.0) \\
0.0-1.1\end{array}$ & $\begin{array}{l}1(0.4) \\
0.0-4.5\end{array}$ & $\begin{array}{l}0(0.0) \\
0.0-0.8\end{array}$ & $\begin{array}{l}1(0.1) \\
0.0-0.7\end{array}$ \\
\hline Hyperhidrosis (10020642) & & $\begin{array}{l}1(0.3) \\
0.0-3.9\end{array}$ & $\begin{array}{l}0(0.0) \\
0.0-1.3\end{array}$ & $\begin{array}{l}0(0.0) \\
0.0-0.8\end{array}$ & $\begin{array}{l}1(0.1) \\
0.0-0.6\end{array}$ \\
\hline $\begin{array}{l}\text { Any ear and labyrinth disorders } \\
(\mathbf{1 0 0 1 3 9 9 3 )}\end{array}$ & $\begin{array}{l}\text { n }(\%) \\
\text { 95\% CI, } \\
\text { LL-UL }\end{array}$ & $\begin{array}{l}\mathbf{1}(\mathbf{0 . 3}) \\
\mathbf{0 . 0}-9.8\end{array}$ & $\begin{array}{l}5(1.8) \\
0.0-10.2\end{array}$ & $\begin{array}{l}1(0.2) \\
0.0-2.3\end{array}$ & $\begin{array}{l}7(0.7) \\
0.1-2.2\end{array}$ \\
\hline $\begin{array}{l}\text { Ear pain (10014020); vertigo } \\
\quad(10047340)\end{array}$ & & $\begin{array}{l}n=0 ; 0 \\
\mathrm{NR}\end{array}$ & $\begin{array}{l}n=1 ; 2 \\
\mathrm{NR}\end{array}$ & $\begin{array}{l}n=1 ; 0 \\
\mathrm{NR}\end{array}$ & $\begin{array}{l}2(0.2) \text { each } \\
\text { NR }\end{array}$ \\
\hline $\begin{array}{l}\text { Ear haemorrhage (10014009); ear } \\
\text { pruritus (10052138); tinnitus } \\
(10043882)\end{array}$ & & $\begin{array}{l}n=0 ; 1 ; 0 \\
\mathrm{NR}\end{array}$ & $\begin{array}{l}n=1 ; 0 ; 1 \\
\mathrm{NR}\end{array}$ & $\begin{array}{l}n=0 ; 0 ; 0 \\
\mathrm{NR}\end{array}$ & $\begin{array}{l}1(0.1) \text { each } \\
\text { NR }\end{array}$ \\
\hline $\begin{array}{l}\text { Any immune system disorders } \\
(\mathbf{1 0 0 2 1 4 2 8})\end{array}$ & $\begin{array}{l}\text { n }(\%) \\
\text { 95\% CI, } \\
\text { LL-UL }\end{array}$ & $\begin{array}{l}1(0.3) \\
0.0-3.9\end{array}$ & $\begin{array}{l}0(0.0) \\
0.0-1.3\end{array}$ & $\begin{array}{l}0(0.0) \\
0.0-0.8\end{array}$ & $\begin{array}{l}1(0.1) \\
0.0-0.6\end{array}$ \\
\hline Hypersensitivity (10020751) & & $\begin{array}{l}1(0.3) \\
0.0-3.9\end{array}$ & $\begin{array}{l}0(0.0) \\
0.0-1.3\end{array}$ & $\begin{array}{l}0(0.0) \\
0.0-0.8\end{array}$ & $\begin{array}{l}1(0.1) \\
0.0-0.6\end{array}$ \\
\hline Anaphylactic reaction (10002198) & & $\begin{array}{l}0(0.0) \\
0.0-1.1\end{array}$ & $\begin{array}{l}0(0.0) \\
0.0-1.3\end{array}$ & $\begin{array}{l}0(0.0) \\
0.0-0.8\end{array}$ & $\begin{array}{l}0(0.0) \\
0.0-0.4\end{array}$ \\
\hline Any investigations (10022891) & $\begin{array}{l}\text { n }(\%) \\
\text { 95\% CI, } \\
\text { LL-UL }\end{array}$ & $\begin{array}{l}1(0.3) \\
0.0-3.9\end{array}$ & $\begin{array}{l}3(1.1) \\
0.0-13.1\end{array}$ & $\begin{array}{l}0(0.0) \\
0.0-0.8\end{array}$ & $\begin{array}{l}4(0.4) \\
0.0-1.8\end{array}$ \\
\hline Heart rate increased (10019303) & & $\begin{array}{l}1(0.3) \\
0.0-3.9\end{array}$ & $\begin{array}{l}3(1.1) \\
0.0-13.1\end{array}$ & $\begin{array}{l}0(0.0) \\
0.0-0.8\end{array}$ & $\begin{array}{l}4(0.4) \\
0.0-1.8\end{array}$ \\
\hline Any eye disorders (10015919) & $\begin{array}{l}\text { n }(\%) \\
\text { 95\% CI, } \\
\text { LL-UL }\end{array}$ & $\begin{array}{l}1(0.3) \\
0.0-9.8\end{array}$ & $\begin{array}{l}3(1.1) \\
0.1-3.7\end{array}$ & $\begin{array}{l}0(0.0) \\
0.0-0.8\end{array}$ & $\begin{array}{l}4(0.4) \\
0.0-1.4\end{array}$ \\
\hline
\end{tabular}


Table 3 (continued)

\begin{tabular}{|c|c|c|c|c|c|}
\hline $\begin{array}{l}\text { MedDRA Primary System Organ } \\
\text { Class (CODE) } \\
\text { Preferred Term (Code) }\end{array}$ & & Belgium $[N=329]$ & Germany $[N=278]$ & Spain $[N=453]$ & Total $[N=1060]$ \\
\hline $\begin{array}{l}\text { Eye discharge (10015915), eye } \\
\text { pruritus (10052140); lacrimation } \\
\text { increased (10023644); swelling of } \\
\text { eyelid (10042690) }\end{array}$ & & $\begin{array}{l}n=0 ; 0 ; 0 ; 1 \\
\mathrm{NR}\end{array}$ & $\begin{array}{l}n=1 ; 1 ; 1 ; 0 \\
\mathrm{NR}\end{array}$ & $\begin{array}{l}n=0 ; 0 ; 0 ; 0 \\
\mathrm{NR}\end{array}$ & $\begin{array}{l}1(0.1) \text { each } \\
\text { NR }\end{array}$ \\
\hline $\begin{array}{l}\text { Any injury, poisoning and proce- } \\
\text { dural complications (10022117) }\end{array}$ & $\begin{array}{l}\text { n }(\%) \\
95 \% \text { CI, } \\
\text { LL-UL }\end{array}$ & $\begin{array}{l}\mathbf{0}(0.0) \\
0.0-1.1\end{array}$ & $\begin{array}{l}1(0.4) \\
0.0-4.5\end{array}$ & $\begin{array}{l}0(0.0) \\
0.0-0.8\end{array}$ & $\begin{array}{l}1(0.1) \\
0.0-0.7\end{array}$ \\
\hline Muscle strain (10050031) & & $\begin{array}{l}0(0.0) \\
0.0-1.1\end{array}$ & $\begin{array}{l}1(0.4) \\
0.0-4.5\end{array}$ & $\begin{array}{l}0(0.0) \\
0.0-0.8\end{array}$ & $\begin{array}{l}1(0.1) \\
0.0-0.7\end{array}$ \\
\hline Any cardiac disorders (10007541) & $\begin{array}{l}\text { n }(\%) \\
\text { 95\% CI, } \\
\text { LL-UL }\end{array}$ & $\begin{array}{l}0(0.0) \\
0.0-1.1\end{array}$ & $\begin{array}{l}1(0.4) \\
0.0-7.2\end{array}$ & $\begin{array}{l}0(0.0) \\
0.0-0.8\end{array}$ & $\begin{array}{l}1(0.1) \\
0.0-0.7\end{array}$ \\
\hline Tachycardia (10043071) & & $\begin{array}{l}0(0.0) \\
0.0-1.1\end{array}$ & $\begin{array}{l}1(0.4) \\
0.0-7.2\end{array}$ & $\begin{array}{l}0(0.0) \\
0.0-0.8\end{array}$ & $\begin{array}{l}1(0.1) \\
0.0-0.7\end{array}$ \\
\hline Any vascular disorders (10047065) & $\begin{array}{l}\text { n }(\%) \\
95 \% \text { CI, } \\
\text { LL-UL }\end{array}$ & $\begin{array}{l}1(0.3) \\
0.0-5.5\end{array}$ & $\begin{array}{l}\text { O }(0.0) \\
0.0-1.3\end{array}$ & $\begin{array}{l}0(0.0) \\
0.0-0.8\end{array}$ & $\begin{array}{l}1(0.1) \\
0.0-0.7\end{array}$ \\
\hline Hot flush (10060800) & & $\begin{array}{l}1(0.3) \\
0.0-5.5\end{array}$ & $\begin{array}{l}0(0.0) \\
0.0-1.3\end{array}$ & $\begin{array}{l}0(0.0) \\
0.0-0.8\end{array}$ & $\begin{array}{l}1(0.1) \\
0.0-0.7\end{array}$ \\
\hline
\end{tabular}

Bold values represent the MedDRA Primary System Organ Class (SOC)

Italic values represent Preferred Terms which corresponds to the pre-defined AEIs

Plain values represent Preferred Terms which corresponds to other AEs captured beyond the initial pre-defined AEIs

NR: AEs that occurred in three or fewer cases were grouped to simplify the table, with the number of cases presented for each symptom and the total number (\%) for each symptom, but not each individual 95\% CI

$A E$ adverse event, $A E I$ adverse event of interest, MedDRA Medical Dictionary for Regulatory Activities, $N$ total number of subjects, $n(\%)$ number (percentage) of subjects reporting the symptom at least once, 95\% CI 95\% confidence interval (extended Clopper-Pearson exact CI for cluster data), $L L$ lower limit, $U L$ upper limit, $N R$ not reported

\section{Discussion}

The design of this enhanced safety study allowed for rapid recruitment and near real-time monitoring of AEs following vaccination with GSK's IIV4, in paediatric, adult and older adult subjects. The study captured all age groups indicated for vaccination with GSK's IIV4 and provided an overall picture of AEs experienced, including in the paediatric population. A strength of this study was the inclusion of subjects from different countries and age groups, which allowed capturing different vaccination practices and diversity in population characteristics.

Nearly all patients completed and returned the AERC ( $98.3 \%$ post dose $1,100 \%$ post dose 2 ). The very high return rates of AERCs, similar to response rates reported in active studies [18], supports the appropriateness of the approach and procedures used to reliably and comprehensively assess the AEs experienced after vaccination. The electronic application used to collect standardised data facilitated data extraction, cleaning and analysis with the objective of maximising near real-time assessment. Therefore, the timely electronic data encoding by the medical team allowed for continuous assessment, e.g. weekly review of AEs, and thus prompt investigation if necessary. Furthermore, ongoing review of events allowed prompt clarification from centres via individual queries.

There were 1060 subjects enrolled from October to November 2018, 1035 of whom completed the study. The number of withdrawals $(n=25)$ was limited and none were due to AEs. Reasons were lost to follow-up for adult subjects $(n=17)$ and consent withdrawal not due to an AE for paediatric subjects $(n=7$, mostly as parents decided not to administer dose 2 of the vaccine). Overall, $43.0 \%$ and $23.7 \%$ of subjects reported at least one AE following dose 1 and dose 2 , respectively. The most frequently reported AEs were as expected on the predefined list (i.e. AEIs), and included mainly two MedDRA SOCs; General Disorders and Administration Site Conditions, and Respiratory, Thoracic and Mediastinal Disorders. No safety signals were detected for GSK's IIV4 during the course of the study or at any intermediate time points (e.g. weekly review of individual data listings, interim analyses) or at the end of the study period (final analysis). No new or unexpected AEs were reported, and all AEs reported were within the expected scope of events and event rates for the populations studied. The incidence 
Table 4 Cumulative subjects (\%) reporting AEIs (italic) and/ or other AEs post dose 2 over the study period (Safety Set)
MedDRA Primary System Organ Class (CODE)

Preferred Term (Code)

Any

Any respiratory, thoracic and mediastinal disorders (10038738)

Cough (10011224)

Rhinorrhoea (10039101)

Nasal congestion (10028735)

Dysphonia (10013952)

Wheezing (10047924)

Epistaxis (10015090)

Sneezing (10041232)

Oropharyngeal pain (10068319)

Any general disorders and administration site conditions (10018065)

Injection site pain (10022086)

Pyrexia (10037660)

Chills (10008531)

Face oedema (10016029)

Fatigue (10016256)

Injection site erythema (10022061) or swelling (10053425)

Any infections and infestations (10021881)

Conjunctivitis (10010741)

Rhinitis (10039083)

Any gastrointestinal disorders (10017947)

Diarrhoea (10012735)

Vomiting (10047700)

Abdominal pain (10000081)

Nausea (10028813)

Any metabolism and nutrition disorders (10027433)

Decreased appetite (10061428)

Any psychiatric disorders (10037175)

Irritability (10022998)

Any eye disorders (10015919)

Eye pruritus (10052140)

Any musculoskeletal and connective tissue disorders (10028395)

Myalgia (10028411)

Arthropathy (10003285)

Any skin and subcutaneous tissue disorders (10040785)

Rash (10037844)

Rash generalized (10037858)

Any immune system disorders (10021428)

Anaphylaxis (10002198) or Hypersensitivity (10020751)

Any nervous system disorders (10029205)

Febrile convulsion (10016284) or Headache (10019211)

Bold values represent the MedDRA Primary System Organ Class (SOC)

Italic values represent Preferred Terms which corresponds to the pre-defined AEIs

Plain values represent Preferred Terms which corresponds to other AEs captured beyond the initial predefined AEIs

$A E$ adverse event, $A E I$ adverse event of interest, MedDRA Medical Dictionary for Regulatory Activities, $N$ total number of subjects, $n(\%)$ number (percentage) of subjects reporting the symptom at least once, $95 \%$ CI 95\% confidence interval (extended Clopper-Pearson exact CI for cluster data), $L L$ lower limit, $U L$ upper limit 
Table 5 Cumulative subjects (\%) reporting AEIs (italic) and/or other AEs post dose 1 and dose 2 over the study period - Spain (Safety Set)

MedDRA Primary System Organ Class (CODE)

Preferred Term (Code)

\begin{tabular}{rrl}
\multicolumn{2}{l}{ Spain $[N=453]$} & \\
\hline$n$ & $\mathbf{n}$ & $\%[95 \%$ CI $(\mathrm{LL}-\mathrm{UL})]$ \\
$\mathbf{6 6 9}$ & $\mathbf{2 2 7}$ & $\mathbf{5 0 . 1}[\mathbf{2 9 . 1}-\mathbf{7 1 . 1}]$ \\
$\mathbf{2 3 3}$ & $\mathbf{1 4 6}$ & $\mathbf{3 2 . 2}[\mathbf{1 1 . 9}-\mathbf{5 9 . 1}]$ \\
83 & 82 & $18.1[2.3-51.5]$ \\
36 & 35 & $7.7[2.2-18.4]$ \\
36 & 34 & $7.5[1.2-22.8]$ \\
33 & 33 & $7.3[1.7-18.9]$ \\
25 & 25 & $5.5[2.4-10.6]$ \\
13 & 13 & $2.9[0.3-10.1]$ \\
1 & 1 & $0.2[0.0-2.3]$ \\
2 & 2 & $0.4[0.1-1.6]$ \\
1 & 1 & $0.2\left[0.0-4.8 / 7.3^{\mathrm{a}}\right]$
\end{tabular}

(10022093); peripheral swelling (10048959) ${ }^{\mathrm{a}}$

Any respiratory, thoracic and mediastinal disorders (10038738)

212

Cough (10011224)

Rhinorrhea (10039101)

Nasal congestion (10028735)

Oropharyngeal pain (10068319)

Dysphonia (10013952)

Epistaxis (10015090)

Wheezing (10047924)

Sneezing (10041232)

1 each: Increased upper airway secretion (10062717); Throat clearing (10080125) ${ }^{\mathrm{b}}$

Any musculoskeletal and connective tissue disorders (10028395)

Myalgia (10028411)

Arthropathy (10003285)

1 each: Pain in extremity (10033425); Synovial cyst $(10042858)^{\mathrm{c}}$

Any nervous system disorders (10029205)

Headache (10019211)

1 each: Aphonia (10002953); Dizziness (10013573); Somnolence (10041349)

Febrile convulsion (10016284)

Any gastrointestinal disorders (10017947)

Diarrhoea (10012735)

Vomiting (10047700)

Nausea (10028813)

Abdominal pain (10000081)

Abdominal discomfort (10000059)

Any infections and infestations (10021881)

Conjunctivitis (10010741)

Rhinitis (10039083)

1 each (n): Bronchitis (10006451); Ear infection (10014011); Hordeolum (10020377)

Laryngitis $(10023874)^{\mathrm{d}}$; Nasopharyngitis $(10028810)^{\mathrm{d}}$; Upper respiratory tract infection (10046306)

Any metabolism and nutrition disorders (10027433)

Decreased appetite (10061428)

Any psychiatric disorders (10037175)

Irritability (10022998)

Mood swings (10027951)

Any skin and subcutaneous tissue disorders (10040785)

Rash (10037844)
$25.6[12.5-42.9]$

$12.6[2.8-32.2]$

$11.5[5.0-21.6]$

$8.8[6.2-12.2]$

$5.7[1.3-15.2]$

$2.9[1.2-5.6]$

1.1 [0.0-5.7]

0.9 [0.2-2.3]

0.7 [0.0-6.9]

$0.2\left[0.0-2.3 / 4.8^{\mathrm{b}}\right]$

8.6 [0.4-35.5]

7.1 [0.5-26.6]

2.4 [0.0-29.2]

$0.2\left[0.0-2.3 / 4.8^{\mathrm{c}}\right]$

8.0 [0.6-29.7]

7.3 [1.1-22.5]

$0.2[0.0-4.8]$

0.0 [0.0-0.8]

7.1 [4.7-10.1]

$4.0[2.4-6.2]$

$3.3[0.2-13.6]$

2.9 [0.3-10.1]

0.9 [0.0-9.1]

$0.2[0.0-4.8]$

7.3 [1.2-22.0]

3.5 [0.3-14.3]

3.1 [0.2-12.9]

$0.2\left[0.0-2.3 / 7.3^{\mathrm{d}}\right]$

$5.7[0.2-26.1]$

5.7 [0.2-26.1]

2.9 [0.8-7.1]

$2.9[0.8-7.1]$

$0.2[0.0-4.8]$

$1.6[0.0-11.4]$

$1.1[0.0-7.8]$ 
Table 5 (continued)

\begin{tabular}{lrrr}
\hline MedDRA Primary System Organ Class (CODE) & \multicolumn{2}{l}{ Spain $[N=453]$} \\
\cline { 2 - 4 } Preferred Term (Code) & $n$ & $\mathbf{n}$ & $\%[95 \%$ CI (LL-UL)] \\
\hline Erythema (10015150), & 2 & 2 & $0.4[0.0-4.0]$ \\
Rash generalized (10037858) & 0 & 0 & $0.0[0.0-0.8]$ \\
Any ear and labyrinth disorders (10013993) & $\mathbf{1}$ & $\mathbf{1}$ & $\mathbf{0 . 2}[\mathbf{0 . 0}-\mathbf{2 . 3}]$ \\
Ear pain (10014020) & 1 & 1 & $0.2[0.0-2.3]$ \\
Any immune system disorders (10021428) & $\mathbf{0}$ & $\mathbf{0}$ & $\mathbf{0 . 0}[\mathbf{0 . 0}-\mathbf{0 . 8}]$ \\
Hypersensitivity (10020751) & 0 & 0 & $0.0[0.0-0.8]$ \\
Anaphylactic reaction (10002198) & 0 & 0 & $0.0[0.0-0.8]$ \\
Any eye disorders (10015919) & $\mathbf{2}$ & $\mathbf{2}$ & $\mathbf{0 . 4}[\mathbf{0 . 0}-\mathbf{4 . 6}]$ \\
Eye pruritus (10052140) & 2 & 2 & $0.4[0.0-4.6]$ \\
\hline
\end{tabular}

Bold values represent the MedDRA Primary System Organ Class (SOC)

Italic values represent Preferred Terms which corresponds to the pre-defined AEIs

Plain values represent Preferred Terms which corresponds to other AEs captured beyond the initial pre-defined AEIs

$A E$ adverse event, $A E I$ adverse event of interest, MedDRA Medical Dictionary for Regulatory Activities, $N$ total number of subjects, $n$ number of specified AEs reported considering both AERCs, $n(\%)$ number (percentage) of subjects reporting the symptom at least once, $95 \%$ CI 95\% confidence interval (extended Clopper-Pearson exact CI for cluster data), $L L$ lower limit, $U L$ upper limit, AERCs adverse event reporting cards

a,b,c,d $95 \%$ CI UL for the specified AEs

rates of AEs were similar in magnitude to those described in GSK's quadrivalent influenza vaccine Summary of Product Characteristics (SmPC) [19].

A recent review summarises the initiatives conducted to date to address the EMA requirement and illustrates the diversity of approaches that can be used to conduct safety surveillance in Europe. This paper also provides an overview of surveillance initiatives outside of Europe and tools that are used to conduct surveillance for seasonal influenza vaccines [5]. In the guidance, several monitoring options were suggested to carry out the surveillance, including active surveillance, enhanced surveillance, and data mining or use of electronic health records (EHRs). The review identified nine enhanced surveillance studies using a variety of tools (e.g. safety reporting cards, daily diaries, telephone interviews, EHR data, text message reminders and web-based questionnaires), combining several tools in some studies. All studies successfully implemented the new methodology and collected near real-time data on AEIs, identifying no safety signals. There were however difficulties identified in some studies, e.g. recruiting sufficient participants to capture less frequent AEs, recruiting participants from all age groups (especially younger participants), or receiving sufficient completed safety reporting cards [5].

A novel approach to rapid vaccine safety surveillance used in Australia involved active SMS-based surveillance. In this study in 2015 and 2016 of 7402 children, an SMS or email was used to survey parents on AEs experienced within 3 days of vaccination with different influenza vaccines, and data were reported weekly. As in our study, AE rates were found to be low and as expected per the SmPC [20].
Our study bears some limitations. For instance, the length of the enrolment period was dependent upon the timing of vaccination campaigns in participating countries and did not allow enrolment to start before mid to end October for most participating sites. Furthermore, the study was not designed/ powered to capture rare AEs, although this was not the objective of the EMA initiative because this will continue to be covered by routine pharmacovigilance activities as per manufacturers' obligations. However, such studies contribute to the continuous monitoring of vaccine safety and complement the routine pharmacovigilance, which has been discussed extensively elsewhere [5]. Vaccine coadministration (on the same day as GSK's IIV4) was captured, however due to the relatively low numbers reported, it was not possible to observe any trends or impact on the frequency of AEs experienced.

\section{Conclusions}

In compliance with the EMA guidance, this enhanced safety study was designed to assess AEIs experienced within 7 days post vaccination with GSK's IIV4. Subjects from all vaccinated age groups were enrolled and AEs were monitored and analysed in near real-time, confirming the suitability of the study methodology used to appropriately monitor the safety of the seasonal influenza vaccine. In this study, no safety signals that impact public health or alter the benefit-risk profile of GSK's IIV4 were identified during the course of the study or at the end of the study period. The study supports 
and confirms the findings were in line with the safety profile of GSK's IIV4 for all age groups the vaccine is indicated for.

Acknowledgements The authors thank the patients for their participation in the study and the medical staff who contributed to the study implementation. In particular, the authors thank Jelena Bakusic, Stephanie Gilon, Katie Smith, Jacqueline Miller, Celine Jegou, Dominique Rosillon, Kusuma Gopala, Sophie Caterina, Frederic Lin, Els Van de Paar, Sonia Sierra Domingez, Gwenny Honee and Stéphane Vanden Bemden for their contribution to this study. The authors also thank Business \& Decision Life Sciences platform for editorial assistance and manuscript coordination, on behalf of GSK. Kavi Littlewood (Littlewood Writing Solutions, on behalf of GSK) provided writing support, and Bruno Dumont coordinated manuscript development and editorial support.

Author Contributions Gael Dos Santos led and wrote the first draft of the manuscript. All authors participated in the design, implementation or analysis, and interpretation of the study, and the development of this manuscript. All authors had full access to the data and gave final approval before submission. The authors are solely responsible for the final content and received no financial support or other form of compensation related to the development of this manuscript. The material is original and has not been submitted elsewhere.

\section{Compliance with Ethical Standards}

Conflicts of Interest Gael Dos Santos, Silvia Damaso, Anne Yeakey and Bach-Yen Nguyen declare they are employed by the GSK group of companies and hold shares in the GSK group of companies. Xavier Martínez Gómez declares having received financial support from PaxVax for participation on advisory boards for vaccines and from Pfizer for participation at conferences. Alexander C. Schmidt declares he was employed by the GSK group of companies at the time of the study conduct and holds shares in the GSK group of companies. Vishvesh Shende declares he is employed by VPN Consultancy Limited, working on behalf of the GSK group of companies. Hugo Loos, Lode Godderis, Tamara Eckermann and Ignacio Salamanca de la Cueva declare they have no conflicts of interest.

Funding GlaxoSmithKline Biologicals SA is the funding source and was involved in all stages of the study, and funded all costs associated with the development and publishing of the present manuscript. All authors had full access to the data and agreed with the submission of the publication.

Ethical Approval All procedures performed in studies involving human participants were in accordance with the ethical standards of the institutional and/or national research committees and with the 1964 Helsinki declaration and its later amendments or comparable ethical standards. The following Ethics Committees (ECs), or Institutional Review Boards (IRBs) and Regional Authorities were consulted in line with country requirements, namely Commissie Medische Ethiek UZ/ KU Leuven (EC) in Belgium (S61698; approval received 24 August); Ethik-Kommission der Bayerischen (EC) in Germany (18056; approval received 20 September); and, in Spain, the PEIBA (IRB and RA combined) for Andalusia (0031/18 EPA-SP; approval received 26 September) and Hospital Vall de Hebron (IRB) and Goli Gurina (RA) for Catalonia (ID-RTF-021; approval received 22 October).

Informed Consent Informed consent was obtained from all individual participants included in this study.
Data Availability To request access to patient-level data and documents for this study, please submit an enquiry via www.clinicalstudydatareq uest.com.

Trademarks AlphaRix Tetra, Influsplit Tetra and Fluarix Tetra are trademarks of the GSK group of companies.

Open Access This article is licensed under a Creative Commons Attribution-NonCommercial 4.0 International License, which permits any non-commercial use, sharing, adaptation, distribution and reproduction in any medium or format, as long as you give appropriate credit to the original author(s) and the source, provide a link to the Creative Commons licence, and indicate if changes were made. The images or other third party material in this article are included in the article's Creative Commons licence, unless indicated otherwise in a credit line to the material. If material is not included in the article's Creative Commons licence and your intended use is not permitted by statutory regulation or exceeds the permitted use, you will need to obtain permission directly from the copyright holder. To view a copy of this licence, visit http://creativecommons.org/licenses/by-nc/4.0/.

\section{References}

1. World Health Organisation (WHO). Fact Sheets. Influenza (seasonal). http://www.who.int/news-room/fact-sheets/detail/influ enza-(seasonal). Accessed 1 Apr 2019.

2. European Centre for Disease Prevention and Control (ECDC). Factsheet about seasonal influenza. https://ecdc.europa.eu/en/ seasonal-influenza/facts/factsheet. Accessed 1 Jul 2019.

3. Foppa IM, Cheng PY, Reynolds SB, Shay DK, Carias C, Bresee JS et al. Deaths averted by influenza vaccination in the U.S. during the seasons 2005/06 through 2013/14. Vaccine. 2015;33(26):3003-9. https://doi.org/10.1016/j.vacci ne.2015.02.042.

4. World Health Organisation (WHO). WHO recommendations on the composition of influenza virus vaccines. https://www.who. int/influenza/vaccines/virus/recommendations/en/. Accessed $1 \mathrm{Jul}$ 2019.

5. Dos Santos G. Challenges in implementing yearly enhanced safety surveillance of influenza vaccination in Europe: lessons learned and future perspectives. Hum Vaccin Immunother. 2019;15(11):2624-36. https://doi.org/10.1080/21645 515.2019 .1608745 .

6. European Medicines Agency (EMA). Explanatory note on the withdrawal of the Note for guidance on harmonisation of requirements for influenza Vaccines and of the core SmPC/PL for inactivated seasonal influenza vaccines. 2014. https://www.ema.europ a.eu/en/documents/scientific-guideline/explanatory-note-withd rawal-note-guidance-harmonisation-requirements-influenza-vacci nes-core-summary_en-0.pdf. Accessed 1 Apr 2019.

7. European Medicines Agency (EMA). Interim guidance on enhanced safety surveillance for seasonal influenza vaccines in the EU 2014. http://www.ema.europa.eu/docs/en_GB/document_libra ry/Scientific_guideline/2014/04/WC500165492.pdf. Accessed 1 Apr 2019.

8. De Lusignan S, Correa A, Dos Santos G, Meyer N, Haguinet F, Webb R, et al. Brand specific, near real time, enhanced safety surveillance of influenza vaccines in general practice winter 2015/16: feasibility study. JMIR Public Health Surveill. 2019;5(4):e12016. https://doi.org/10.2196/12016.

9. De Lusignan S, Ferreira F, Damaso S, Byford R, Pathirannehelage S, Yeakey A, et al. Enhanced passive surveillance of influenza vaccination in England, 2016-2017: an observational 
study using an adverse events reporting card. Hum Vaccin Immunother. 2019;15(5):1048-59. https://doi.org/10.1080/21645 515.2019.1565258.

10. de Lusignan S, Dos Santos G, Byford R, Schuind A, Damaso S, Shende V, et al. Enhanced safety surveillance of seasonal quadrivalent influenza vaccines in english primary care: interim analysis. Adv Ther. 2018;35(8):1199-214. https://doi.org/10.1007/ s12325-018-0747-4.

11. Dos Santos G, Shende V, Damaso S, Yeakey A. Enhanced safety surveillance of GSK's quadrivalent seasonal influenza vaccine in Belgium, Germany and Spain for the 2018/19 season: interim analysis. Adv Ther. 2019;36:3340-55.

12. De Lusignan S, Damaso S, Ferreira F, Byford R, McGee C, Pathirannehelage $S$ et al. UNDER REVIEW MANUSCRIPT: Brandspecific Enhanced Safety Surveillance of GSK's Fluarix Tetra seasonal influenza vaccine in England: 2017/2018 season. 2019.

13. Dos Santos G, Yeakey A, Shende V, Smith K, Lin F, Zandmanvan Dijk E, et al. Passive enhanced safety surveillance of GSK's quadrivalent seasonal influenza vaccine in Belgium, Germany and Spain, an observational study: protocol for the 2018/2019 influenza season. BMJ Open. 2019;9:e028043. https://doi.org/10.1136/ bmjopen-2018-028043.

14. European Centre for Disease Prevention and Control (ECDC). Vaccine schedules in all countries of the European Union. 2018. https://vaccine-schedule.ecdc.europa.eu/. Accessed 1 Jul 2019.
15. Medical Dictionary for Regulatory Activities MedDRA. https:// www.meddra.org/basics. Accessed 1 Apr 2019.

16. ClinicalTrials.gov. Enhanced Safety Surveillance of GlaxoSmithKline's (GSK's) Quadrivalent Seasonal Influenza Vaccines During the 2018/19 Influenza Season. 2019. https://clinicaltrials.gov/ct2/ show $/$ NCT03688620?term $=$ NCT03688620\&rank $=1$. Accessed 2 Sep 2019

17. GSK. GSK Study Register. 2019. https://www.gsk-studyregister. com/study/6027. Accessed 2 Sep 2019.

18. Spila Alegiani S, Alfonsi V, Appelgren EC, Ferrara L, Gallo $\mathrm{T}$, Alicino $\mathrm{C}$, et al. Active surveillance for safety monitoring of seasonal influenza vaccines in Italy, 2015/2016 season. BMC Public Health. 2018;18(1):1401. https://doi.org/10.1186/s1288 9-018-6260-5.

19. Medicines.org.uk. Fluarix Tetra Summary of Product Characteristics (SmPC). 2018. https://www.medicines.org.uk/emc/produ ct/3021/smpc. Accessed 18 May 2019.

20. Pillsbury A, Quinn H, Cashman P, Leeb A, Macartney K. Active SMS-based influenza vaccine safety surveillance in Australian children. Vaccine. 2017;35(51):7101-6. https://doi.org/10.1016/j. vaccine.2017.10.091.

\section{Affiliations}

\section{Gael Dos Santos ${ }^{1}$ [D $\cdot$ Bach-Yen Nguyen ${ }^{2}$. Silvia Damaso ${ }^{1}$ (1) - Lode Godderis ${ }^{3,4} \cdot$ Xavier Martínez-Gómez $^{5}$. Tamara Eckermann ${ }^{6} \cdot$ Hugo Loos $^{7} \cdot$ Ignacio Salamanca de la Cueva ${ }^{8} \cdot$ Vishvesh Shende $^{9}$ (i). Alexander C. Schmidt ${ }^{2,10}$ (i) Anne Yeakey ${ }^{2}$ (i)}

1 GSK, Wavre, Belgium

2 GSK, Rockville, MD, USA

3 Environment and Health, Department of Public Health and Primary Care, KU Leuven, Louvain, Belgium

4 IDEWE Occupational Health Services, Heverlee-Leuven, Belgium

5 Hospital Universitari Vall d'Hebron, Barcelona, Spain

6 General Practitioner, Munich, Germany
7 General Practitioner, Geel, Belgium

8 Instituto Hispalense de Pediatria, Seville, Spain

$9 \quad$ VPN Consultancy Ltd (On Behalf of GSK), London, UK

10 Present Address: Gates Medical Research Institute, Cambridge, MA, USA 